\title{
ON A CONVERSE OF ABEL'S THEOREM
}

\section{AMNON AMIR (JAKIMOVSKI)}

1. Introduction. It is known that if the real power series $\sum_{n=0}^{\infty} a_{n} \cdot x^{n}$ is of radius 1 and $\sum_{n=0}^{\infty} a_{n}$ is summable $(C, \alpha), \alpha>-1$, to $s$, then $\lim _{x+1} \sum_{n=0}^{\infty} a_{n} \cdot x^{n}=s$. This theorem is generalized and stated for the $(A, \alpha)$ methods of summability, which are defined in this paper.

The following general converse of Abel's theorem was given by R. Schmidt: If $\sum_{n=0}^{\infty} a_{n}$ is summable Abel and

$$
\lim _{\lambda \downarrow 1} \lim _{n \rightarrow \infty} \inf \min _{m \leqq n \leqq \lambda \cdot m}\left(s_{n}-s_{m}\right) \geqq 0,
$$

then $\left(s_{n}\right)$ is convergent. A converse of the generalization of Abel's theorem is proved here. This theorem contains Schmidt's theorem as a special case.

Hardy and Littlewood proved that if the sequence $\left(s_{n}\right)$ is summable Abel and for some $\alpha, \alpha>-1$, the $(C, \alpha)$ means of $\left(s_{n}\right)$ are non-negative, then $\left(s_{n}\right)$ is summable $(C, \alpha+1)$. We shall refer in this paper to the special case $\alpha=0$ of this theorem as Theorem $\mathrm{H}$. We shall see that Hardy and Littlewood's theorem follows from Theorem $\mathrm{H}$ and some general theorems for the $(A, \alpha)$ methods of summability. If we suppose in Hardy and Littlewood's theorem the two-sided boundedness of the $(C, \alpha)$ means, then we have more information about the $(C, \beta)$ means.

We shall see that a tauberien theorem for the Nörlund methods of summability follows from Schmidt's theorem.

The author of this paper would like to remark that the results of the original manuscript were less general than those given here. The results of the present paper are an outgrowth of modifications suggested by the referee of the original manuscript.

2. The $(A, \alpha)$ methods of summability. Let $\left(s_{n}\right), n=0,1,2, \cdots$, be an infinite sequence of real numbers. $\left\{\sigma_{n}^{(\alpha)}\right\}$ will be the $(C, \alpha)$ means, $\alpha>-1$, of the sequence $\left(s_{n}\right)$. That is,

$$
\begin{gathered}
\sigma_{n}^{(0)}=s_{n}, \quad \sigma_{n}^{(\alpha)}=\left(C_{n+\alpha, n}\right)^{-1} \cdot \sum_{l=0}^{n} C_{n-l+\alpha-1, n-l} \cdot s_{l}, \\
C_{n+\alpha, n}=\left(1+\frac{\alpha}{1}\right) \cdots\left(1+\frac{\alpha}{n}\right)=\frac{\Gamma(n+\alpha+1)}{\Gamma(n+1) \cdot \Gamma(\alpha+1)}, \alpha>-1 .
\end{gathered}
$$

We shall define $s_{n}^{(\alpha)}$ by

Received by the editors June 1, 1950 and, in revised form, August 2, 1951. 


$$
s_{n}^{(\alpha)}=C_{n+\alpha, n} \cdot \sigma_{n}^{(\alpha)} .
$$

It is easy to see that if $\sum_{n=0}^{\infty} s_{n} \cdot x^{n}$ is convergent for $0 \leqq x<1$, then

$$
(1-x) \cdot \sum_{n=0}^{\infty} s_{n} \cdot x^{n}=(1-x)^{(\alpha+1)} \cdot \sum_{n=0}^{\infty} s_{n}^{(\alpha)} \cdot x^{n}, \quad \alpha>-1 .
$$

We shall prove the following theorem:

TheOREM 2.1. If for some $\alpha, \alpha>-1, \lim _{x+1}(1-x) \cdot \sum_{n=0}^{\infty} \sigma_{n}^{(\alpha)} \cdot x^{n}=s$, then for any $\beta, \beta>\alpha, \lim _{x+1}(1-x) \cdot \sum_{n=0}^{\infty} \sigma_{n}^{(\beta)} \cdot x^{n}=s$.

The special case $\alpha=0$ of Theorem (2.1) is used and proved, without mentioning it explicitly, in proving Hardy and Littlewood's theorem, mentioned in the introduction. The proof of this special case of Theorem (2.1) is given in Titchmarsh's The theory of functions, p. 242, example 8 .

For the proof of Theorem (2.1) we shall need the following lemmas:

Lемма 1. Let $f(x), \phi(x)$ be real integrable functions defined for $0 \leqq x<1, \phi(x)>0$. Let $g(x, t)$ be a real integrable function with a constant sign, defined for $0 \leqq t<x<1$. If $f(x)=o\{\phi(x)\}, \lim _{x+1} \int_{0}^{x} \phi(t) \cdot g(x, t) d t$ $=+\infty$ or $-\infty$, then $\int_{0}^{x} f(t) \cdot g(x, t) d t=o\left\{\int_{0}^{x} \phi(t) \cdot g(x, t) d t\right\}$ for $x \uparrow 1$.

Proof. We may suppose that $g(x, t)$ is positive. For any $\epsilon, \epsilon>0$, there exists a number $x_{0}, 0 \leqq x_{0}<1$, so that for any $t, x_{0} \leqq t<1$, we have $|f(t)| \leqq \epsilon \cdot \phi(t)$. Now for $x_{0} \leqq x<1$

$$
\int_{0}^{x} f(t) \cdot g(x, t) \cdot d t=\left\{\int_{0}^{x_{0}}+\int_{x_{0}}^{x}\right\} f(t) \cdot g(x, t) \cdot d t .
$$

Therefore

$$
\begin{aligned}
\left|\int_{0}^{x} f(t) \cdot g(x, t) \cdot d t\right| & \leqq\left|\int_{0}^{x_{0}} f(t) \cdot g(x, t) \cdot d t\right|+\int_{x_{0}}^{x}|f(t)| \cdot g(x, t) \cdot d t \\
& \leqq A+\epsilon \cdot \int_{0}^{x} \phi(t) \cdot g(x, t) \cdot d t .
\end{aligned}
$$

Since $\int_{0}^{x} \phi(t) \cdot g(x, t) \cdot d t \rightarrow \infty$ for $x \uparrow 1$, there exists a number $x_{1}$, $x_{0} \leqq x_{1}<1$, so that for any $x, x_{1} \leqq x<1, A \leqq \epsilon \cdot \int_{0}^{x} \phi(t) \cdot g(x, t) \cdot d t$, and we have

$$
\left|\int_{0}^{x} f(t) \cdot g(x, t) \cdot d t\right| \leqq 2 \epsilon \cdot \int_{0}^{x} \phi(t) \cdot g(x, t) \cdot d t \quad \text { for } x_{1} \leqq x<1 .
$$

Q.E.D. 
Lemma 2. Let $\alpha$ and $\beta$ be real numbers such that $\beta>\alpha>-1$. If $\sum_{n=0}^{\infty} \sigma_{n}^{(\alpha)} \cdot x^{n}$ is convergent in the unit circle, then there exists a real nonnegative function $g(x, t: \alpha, \beta)$ defined for $0 \leqq t<x<1$, such that we have $\sum_{n=0}^{\infty} \sigma_{n}^{(\beta)} \cdot x^{n+\beta}=\int_{0}^{x} g(x, t: \alpha, \beta) \cdot \sum_{n=0}^{\infty} \sigma_{n}^{(\alpha)} \cdot t^{n+\alpha} \cdot d t ; g(x, t: \alpha, \beta)$ is given by:

(i) Let $\alpha$ be a non-integral real number greater than $-1 . m$ is an integer defined by $m>\alpha>m-1$. If $\beta$ is any real number such that $\beta>\alpha, \beta>0$, then

$$
\begin{aligned}
g(x, t: \alpha, \beta)= & (-1)^{m} \cdot \beta \cdot \Gamma(m-\alpha)^{-1} \cdot \Gamma(\alpha+1)^{-1} \\
& \cdot \frac{d^{m}}{d t^{m}} \int_{t}^{x}(u-t)^{m-\alpha-1} \cdot(x-u)^{\beta-1} \cdot(1-u)^{\alpha-\beta} \cdot d u \\
= & \frac{\Gamma(\beta+1)}{\Gamma(\beta-\alpha) \cdot \Gamma(m-\alpha) \cdot \Gamma(\alpha+1)} \cdot\left(\frac{1-x}{1-t}\right)^{m} \\
& \frac{1}{x-t} \cdot \sum_{n=0}^{\infty} \frac{\Gamma(n+m-\alpha)}{\Gamma(n+1)} \cdot\left(\frac{x-t}{1-t}\right)^{n+\beta-\alpha} .
\end{aligned}
$$

(ii) Let $\alpha$ be a real number such that $0>\alpha>-1$. If $\beta=0$, then

$$
g(x, t: \alpha, \beta)=\Gamma(-\alpha)^{-1} \cdot \Gamma(\alpha+1)^{-1} \cdot(1-x)^{\alpha} \cdot(x-t)^{-\alpha-1} .
$$

(iii) Let $\alpha$ be a real number such that $0>\alpha>-1$. If for $\beta$ we have $0>\beta>\alpha>-1$, then

$$
\begin{aligned}
g(x, t: \alpha, \beta)= & \Gamma(\alpha+1)^{-1} \cdot \Gamma(-\alpha)^{-1} \\
& \cdot \frac{d}{d x} \int_{t}^{x}(u-t)^{-\alpha-1} \cdot(x-u)^{\beta} \cdot(1-u)^{\alpha-\beta} \cdot d u \\
= & \frac{\Gamma(\beta+1)}{\Gamma(\alpha+1) \cdot \Gamma(-\alpha) \cdot \Gamma(\beta-\alpha)} \\
& \cdot \sum_{n=0}^{\infty} \frac{\Gamma(n-\alpha)}{\Gamma(n+1)} \cdot \frac{(x-t)^{n+\beta-\alpha-1}}{(1-t)^{n+\beta-\alpha}} .
\end{aligned}
$$

(iv) Let $\alpha$ be a non-negative integer. If $\beta>\alpha$, then

$$
\begin{aligned}
g(x, t: \alpha, \beta) & =(-1)^{\alpha} \cdot \beta \cdot \Gamma(\alpha+1)^{-1} \cdot \frac{d^{\alpha}}{d t^{\alpha}}\left\{(x-t)^{\beta-1} \cdot(1-t)^{\alpha-\beta}\right\} \\
& =\frac{\Gamma(\beta+1)}{\Gamma(\beta-\alpha) \cdot \Gamma(\alpha+1)} \cdot\left(\frac{1-x}{1-t}\right)^{\alpha} \cdot\left(\frac{x-t}{1-t}\right)^{\beta-\alpha} \cdot \frac{1}{x-t} .
\end{aligned}
$$

Proof. We shall prove (i). The proof of (ii), (iii), and (iv) is similar. By the transformation $u=x-v$ we have 


$$
\begin{aligned}
\int_{t}^{x} & (u-t)^{m-\alpha-1} \cdot(x-u)^{\beta-1} \cdot(1-u)^{\alpha-\beta} \cdot d u \\
& =(1-t)^{\alpha-\beta} \cdot \int_{0}^{x-t}(x-t-v)^{\beta-1} \cdot v^{m-\alpha-1} \cdot\left(1-\frac{v}{1-t}\right)^{\alpha-\beta} \cdot d v \\
& =\sum_{n=0}^{\infty} C_{n+\beta-\alpha-1, n} \cdot(1-t)^{\alpha-\beta-n} \cdot \int_{0}^{x-t}(x-t-v)^{\beta-1} \cdot v^{m+n-\alpha-1} \cdot d v \\
= & \sum_{n=0}^{\infty} \frac{\Gamma(n+\beta-\alpha) \cdot \Gamma(\beta) \cdot \Gamma(m+n-\alpha)}{\Gamma(n+1) \cdot \Gamma(\beta-\alpha) \cdot \Gamma(m+n+\beta-\alpha)} \\
& \cdot(1-t)^{\alpha-\beta-n} \cdot(x-t)^{m+n+\beta-\alpha-1} .
\end{aligned}
$$

We have

$$
\begin{aligned}
& \frac{d^{m}}{d t^{m}}\left\{\frac{(x-t)^{m+n+\beta-\alpha-1}}{(1-t)^{n+\beta-\alpha}}\right\} \\
& =\sum_{k=0}^{m} C_{m, k} \cdot \frac{d^{k}}{d t^{k}}\left\{(x-t)^{m+n+\beta-\alpha-1}\right\} \cdot \frac{d^{m-k}}{d t^{\prime}-k}\left\{\frac{1}{(1-t)^{n+\beta-\alpha}}\right\} \\
& =\frac{\Gamma(m+n+\beta-\alpha)}{\Gamma(n+\beta-\alpha)} \cdot \frac{1}{x-t} \cdot\left(\frac{x-t}{1-t}\right)^{n+m+\beta-\alpha} \cdot \sum_{k=0}^{m} C_{m, k} \cdot\left(-\frac{1-t}{x-t}\right)^{k} \\
& =(-1)^{m} \cdot \frac{\Gamma(m+n+\beta-\alpha)}{\Gamma(n+\beta-\alpha)} \cdot(1-x)^{m} \\
& \cdot \frac{1}{x-t} \cdot\left(\frac{x-t}{1-t}\right)^{n+\beta-\alpha} \cdot \frac{1}{(1-t)^{m}}
\end{aligned}
$$

so that

$$
\begin{aligned}
\frac{d^{m}}{d t^{m}} \int_{t}^{x}(u-t)^{m-\alpha-1} \cdot(x-u)^{\beta-1} \cdot(1-u)^{\alpha-\beta} \cdot d u \\
=(-1)^{m} \cdot \sum_{n=0}^{\infty} \frac{\Gamma(n+\beta-\alpha) \cdot \Gamma(\beta) \cdot \Gamma(m+n+\beta-\alpha) \cdot \Gamma(m+n-\alpha)}{\Gamma(n+1) \cdot \Gamma(\beta-\alpha) \cdot \Gamma(m+n+\beta-\alpha) \cdot \Gamma(n+\beta-\alpha)} \\
\quad \frac{1}{x-t} \cdot(1-x)^{m} \cdot \frac{(x-t)^{n+\beta-\alpha}}{(1-t)^{n+m+\beta-\alpha}} \\
=(-1)^{m} \cdot \frac{\Gamma(\beta)}{\Gamma(\beta-\alpha)} \cdot\left(\frac{1-x}{1-t}\right)^{m} \cdot \frac{1}{x-t} \\
\cdot \sum_{n=0}^{\infty} \frac{\Gamma(n+m-\alpha)}{\Gamma(n+1)} \cdot\left(\frac{x-t}{1-t}\right)^{n+\beta-\alpha} .
\end{aligned}
$$

By partial integration it follows now that 


$$
\begin{aligned}
& \int_{0}^{x}\left\{\sum_{n=0}^{\infty} \sigma_{n}^{(\alpha)} \cdot t^{n+\alpha}\right\} \\
& \cdot \frac{d^{m}}{d t^{m}}\left\{\int_{t}^{x}(u-t)^{m-\alpha-1} \cdot(x-u)^{\beta-1} \cdot(1-u)^{\alpha-\beta} \cdot d u\right\} d t \\
&=(-1)^{m} \cdot \int_{0}^{x} \frac{d^{m}}{d t^{m}}\left\{\sum_{n=0}^{\infty} \sigma_{n}^{(\alpha)} \cdot t^{n+\alpha}\right\} \\
& \cdot\left\{\int_{t}^{x}(u-t)^{m-\alpha-1} \cdot(x-u)^{\beta-1} \cdot(1-u)^{\alpha-\beta} \cdot d u\right\} d t \\
&=(-1)^{m} \cdot \Gamma(m-\alpha) \cdot \Gamma(\alpha+1) \\
& \cdot \int_{0}^{x}(x-u)^{\beta-1} \cdot(1-u)^{\alpha-\beta} \cdot\left\{\sum_{n=0}^{\infty} s_{n}^{(\alpha)} \cdot u^{n}\right\} d u \\
&=(-1)^{m} \cdot \Gamma(m-\alpha) \cdot \Gamma(\alpha+1) \cdot \int_{0}^{x}(x-u)^{\beta-1} \cdot \sum_{n=0}^{\infty} s_{n}^{(\beta)} \cdot u^{n} \cdot d u \\
&=(-1)^{m} \cdot \beta^{-1} \cdot \Gamma(\alpha+1) \cdot \Gamma(m-\alpha)^{\prime} \cdot \sum_{n=0}^{\infty} \sigma_{n}^{(\beta)} \cdot x^{n+\beta} \cdot
\end{aligned}
$$

Q.E.D.

Proof of Theorem 2.1. We may suppose that $s=0$, so that $\sum_{n=0}^{\infty} \sigma_{n}^{(\alpha)} \cdot x^{n+\alpha}=o\left(x^{\alpha} /(1-x)\right)$. If $s_{n}=1$, for $n=0,1,2, \cdots$, then for any $\alpha>-1, \sigma_{n}^{(\alpha)}=1$. By Lemma 1 and Lemma 2 follows now Theorem 2.1 .

It is natural now to define the following methods of summability:

Definition. The sequence $\left(s_{n}\right)$ is summable $(A, \alpha)$ for some $\alpha>-1$, if the power series $\sum_{n=0}^{\infty} s_{n} \cdot x^{n}$ is convergent for $0 \leqq x<1$ and $\lim _{x+1}(1-x) \cdot \sum_{n=0}^{\infty} \sigma_{n}^{(\alpha)} \cdot x^{n}=s$.

From Theorem 2.1 follows:

Theorem 2.2. If the sequence $\left(s_{n}\right)$ is summable $(A, \alpha)$, then it is summable $(A, \beta)$ for any $\beta$ greater than $\alpha$.

THEOREM 2.3. If the sequence $\left(s_{n}\right)$ is summable $(C, \beta)$ for some $\beta>-1$, then it is summable $(A, \alpha)$ for any $\alpha>-1$.

Proof. If $\alpha=\beta$, then the theorem follows from the corresponding theorem for Abel's summability. If $\alpha>\beta$, then $\left(s_{n}\right)$ is also summable $(C, \alpha)$ and the theorem follows from the first part of the proof. If $\alpha<\beta$, then by a well known theorem the sequence $\left(\sigma_{n}^{(\alpha)}\right)$ is summable $(C, \beta-\alpha)$, and the theorem follows from the first part of the proof. 
I have not yet found a sequence which is summable $(A, \beta)$ and is not summable $(A, \alpha)$ if $\alpha<\beta$, but we have the following theorem:

THEOREM 2.4. If for some $\alpha, \alpha>-1, \sigma_{n}^{(\alpha)} \geqq 0, n=0,1,2, \cdots$, and $\left(s_{n}\right)$ is summable $(A, \beta)$ for some $\beta>-1$, then $\left(s_{n}\right)$ is summable $(A, \gamma)$ for any $\gamma>-1$.

Proof. Let $\delta$ be equal to $\max (\alpha, \beta)$, then by a well known theorem it follows that $\sigma_{n}^{(\delta)} \geqq 0$ for $n=0,1,2, \ldots$. Since $\left(s_{n}\right)$ is summable $(A, \delta)$, it follows from Theorem $\mathrm{H}$ that $\left(\sigma_{n}^{(\delta)}\right)$ is summable $(C, 1)$, so that $\left(s_{n}\right)$ is summable $(C, \delta+1)$, and from Theorem (2.3) the proof of this theorem follows.

As a corollary of Theorem 2.4 we have:

Corollary. If for some $\alpha, \alpha>-1, \sigma_{n}^{(\alpha)}>-K, n=0,1,2, \cdots$, and $\left(s_{n}\right)$ is summable $(A, \beta)$ for some $\beta>-1$, then $\left(s_{n}\right)$ is summable $(A, \gamma)$ for any $\gamma>-1$.

TheOREM 2.5 (Hardy and Littlewood's theorem). Let $\alpha$ be some real number such that $\alpha>-1$. If $\left(s_{n}\right)$ is summable Abel and $\sigma_{n}^{(\alpha)}>-K$, for $n=0,1,2, \cdots$, then the sequence $\left(s_{n}\right)$ is summable $(C, \alpha+1)$.

Proof. By the corollary of Theorem 2.4 it follows that $\left(s_{n}\right)$ is summable $(A, \alpha)$. By Theorem $\mathrm{H}$ we see that $\left(\sigma_{n}^{(\alpha)}\right)$ is summable $(C, 1)$, so that $\left(s_{n}\right)$ is summable $(C, \alpha+1)$. Q.E.D.

3. Tauberian theorems for the $(A, \alpha)$ methods of summability.

TheOREM 3.1. If the sequence $\left(s_{n}\right)$ is summable $A$ and

$$
\lim \lim \inf \min _{\lambda \downarrow 1}\left(\sigma_{n}^{(\alpha)}-\sigma_{m}^{(\alpha)}\right) \geqq 0
$$

for some $\alpha, 0 \geqq \alpha>-1$, then the sequence $\left(s_{n}\right)$ is summable $(C, \alpha)$.

In order to prove this theorem we shall need the following lemmas:

Lemma 1. If $N(x), M(x)$ are non-negative integers and $\lim _{x \uparrow 1}(1-x)$ $\cdot N(x)=0, \lim _{x+1}(1-x) \cdot M(x)=+\infty$, then for any $\alpha, 0 \geqq \alpha>-1$,

(i) $\lim _{x+1}(1-x)^{\alpha+1} \cdot \sum_{n=0}^{N(x)} C_{n+\alpha, n} x^{n}=0$,

(ii) $\lim _{x+1}(1-x)^{\alpha+1} \cdot \sum_{n=M(x)}^{\infty} C_{n+\alpha, n} x^{n}=0$,

(iii) $\lim _{x+1}(1-x)^{\alpha+1} \cdot \sum_{n=N(x)}^{M(x)} C_{n+\alpha, n} x^{n}=1$.

Proof. Since

we have

$$
n^{-\alpha} \cdot C_{n+\alpha, n} \rightarrow \frac{1}{\Gamma(\alpha+1)} \quad \text { for } n \uparrow \infty,
$$




$$
\begin{aligned}
(1-x)^{\alpha+1} \cdot \sum_{n=1}^{N(x)} C_{n+\alpha, n} \cdot x^{n} & \leqq A \cdot(1-x)^{\alpha+1} \cdot \sum_{n=1}^{N(x)} n^{\alpha} \\
& \leqq B \cdot\{(1-x) \cdot N(x)\}^{\alpha+1}
\end{aligned}
$$

and (i) follows.

We shall write $\alpha=\beta-1$ so that $1 \geqq \beta>0$. Now

$$
\begin{aligned}
(1-x)^{\alpha+1} \cdot \sum_{n=M(x)}^{\infty} C_{n+\alpha, n} \cdot x^{n} & \leqq A \cdot(1-x)^{\beta} \cdot \sum_{n=M(x)}^{\infty} n^{\alpha} \cdot x^{n} \\
& =A \cdot(1-x)^{\beta} \cdot \sum_{n=M}^{\infty} \frac{x^{n}}{n^{1-\beta}}
\end{aligned}
$$

By the inequality $n^{\beta-1} \leqq C_{1} \cdot(n-M)^{\beta-1} \leqq C_{2} \cdot C_{n-M-\beta+1, n-M}$ for $n>M$ and $M^{\beta-1}<C_{2} \cdot C_{1-\beta, 0}$ it follows that

$$
\begin{aligned}
(1-x)^{\alpha+1} \cdot \sum_{n=M(x)}^{\infty} C_{n+\alpha, n} \cdot x^{n} & \leqq D \cdot x^{M} \cdot(1-x)^{\beta} \cdot \sum_{n=0}^{\infty} C_{n-\beta+1, n} \cdot x^{n} \\
& =D \cdot x^{M} \leqq D \cdot e^{-M \cdot(1-x)}
\end{aligned}
$$

and (ii) follows.

By (i) and (ii), (iii) follows immediately.

Lemma 2. For any positive integers $m, n$ such that $n>m>2$ and for any positive $x$, such that $m \cdot(1-x)>2$, we have $\ln (n / m)<\left(1 / x^{1 / 2}\right)^{n}$.

Proof. From $m \cdot(1-x)>2$ it follows that $0<x<1-2 / m$ $<(1-1 / m)^{2}$, so that $0<x^{1 / 2}<1-1 / m$. Now $1 / x^{1 / 2}>(1-1 / m)^{-1}>1$ $+1 / m$ and $\left(1 / x^{1 / 2}\right)^{n}>(1+1 / m)^{n}>n / m>\ln (n / m)$. Q.E.D.

LEMMA 3. If $0 \geqq \alpha>-1,0 \leqq x<1$, and $M(x)$ is an integer such that $M(x) \cdot(1-x)>2$, and $\lim _{x \uparrow 1}(1-x) \cdot M(x)=+\infty$, then

$$
\lim _{x+1}(1-x)^{\alpha+1} . \sum_{n=M(x)}^{\infty}\left(\ln \frac{n}{m}\right) \cdot C_{n+\alpha, n} \cdot x^{n}=0 .
$$

Proof. By Lemma 2 we have

$$
\begin{aligned}
(1-x)^{\alpha+1} \cdot \sum_{n=M(x)}^{\infty} \ln \frac{n}{m} & \cdot C_{n+\alpha, n} \cdot x^{n} \\
& \leqq(1-x)^{\alpha+1} \cdot \sum_{n=M}^{\infty} C_{n+\alpha, n} \cdot\left(x^{1 / 2}\right)^{n} \\
& \leqq 2^{\alpha+1} \cdot\left(1-x^{1 / 2}\right)^{\alpha+1} \cdot \sum_{n=M(x)}^{\infty} C_{n+\alpha, n} \cdot\left(x^{1 / 2}\right)^{n}
\end{aligned}
$$


For $0 \leqq x<1$ we have $\left(1-x^{1 / 2}\right) \cdot(1-x)^{-1}>1 / 2$ so that $\lim _{x+1}\left(1-x^{1 / 2}\right)$ $\cdot M(x)=+\infty$ and the proof follows by (ii) of Lemma 1 .

LEMmA 4. Let $\left(s_{n}\right)$ be a sequence of real numbers such that $\lim _{\lambda \downarrow 1} \lim \inf _{m \rightarrow \infty} \min _{m \leqq n \leqq \lambda \cdot m}\left\{s_{n}-s_{m}\right\} \geqq 0$, then for any positive constant $c$ there exists a non-negative constant $k(c)$ such that for any positive integers $m, n, m<n$, we have

$$
s_{n}-s_{m}>-(k \cdot \ln n / m+c) \text {. }
$$

Proof. If $s_{n}-s_{m}>-c$ for any $n>m$, then $k(c)=0$. If this does not happen, then there exists a constant $a=a(c), a>0$, so that for any positive integers $m, n$ such that $m>m_{0}(c)$ and $1<n / m \leqq 1+2 a$, we have $s_{n}-s_{m}>-c$. Let $r$ be a non-negative integer defined by $m$ $\cdot(1+a)^{r}<n \leqq m \cdot(1+a)^{r+1}$, then

$$
\begin{aligned}
s_{n}-s_{m}= & \left\{s_{n}-s_{m \cdot(1+a)^{r}}\right\}+\left\{s_{m \cdot(1+a)^{r}}-s_{m \cdot(1+a)^{r-1}}\right\}+\cdots \\
& +\left\{s_{m \cdot(1+a)}-s_{m}\right\}
\end{aligned}
$$

where $s_{m \cdot(1+a)^{p}}$ stands for $s_{\left[m \cdot(1+a)^{p}\right]}$. Let $m$ be greater than $(1+2 a)$ $\cdot a^{-1}$, then $\left[m \cdot(1+a)^{p+1}\right] /\left[m \cdot(1+a)^{p}\right] \leqq 1+2 a$, and from the inequality $m \cdot(1+a)^{r}<n$ follows the inequality $r+1<(1 / \ln (1+a))$ $\cdot \ln (n / m)+1$, so that for $m>\max \left((1+2 a) / a, m_{0}\right)$ we have

$$
s_{n}-s_{m}>-\left(r+1 \cdot c>-\left\{\frac{c}{\ln (1+a)} \cdot \ln \frac{n}{m}+c\right\}\right.
$$

and the lemma is true for $n>m>\max \left((1+2 a / a), m_{0}\right)$. It is easy to see that for a suitable change in the value of the constant $c / \ln (1+a)$ the lemma will be true for any $n>m$.

Proof of Theorem 3.1. In the first part we shall show that the sequence $\left(\sigma_{n}^{(\alpha)}\right)$ is bounded, and in the second part that the same sequence is convergent.

Let us suppose that $\left(\sigma_{n}^{(\alpha)}\right)$ is not bounded, that is, we have $\lim \sup _{m \rightarrow \infty}\left|\sigma_{m}^{(\alpha)}\right|=+\infty$. We cannot have $\lim _{m \rightarrow \infty} \sigma_{m}^{(\alpha)}=+\infty$ or $-\infty$ since then we shall have $\lim _{x \uparrow 1}(1-x) \cdot \sum_{n=0}^{\infty} s_{n} \cdot x^{n}=+\infty$ or $-\infty$, so that $\left(\sigma_{n}^{(\alpha)}\right)$ oscillates between $+\infty$ and $-\infty$.

We shall write $s_{1}(n)=\max _{r \leqq n} \sigma_{r}^{(\alpha)} ; s_{2}(n)=\max _{r \leqq n}\left(-\sigma_{r}^{(\alpha)}\right)$. It is easy to see that for $s_{1}(n), s_{2}(n)$ we may have either

(a) There exists an infinite sequence of positive integers $n_{\nu}, n_{\nu} \rightarrow \infty$, such that $s_{1}\left(n_{v}\right) \geqq s_{2}\left(n_{v}\right)$;

or

(b) For any positive integers $n$ greater than some $n_{0}, s_{1}(n)<s_{2}(n)$, and there is no other possibility. 
Case (a). The functions $s_{1}(n), s_{2}(n)$ are nondecreasing, so that by (a) we have $s_{1}(n) \rightarrow \infty$ for $n \rightarrow \infty$. Let $H>0$, then there exist positive integers $N$ such that $\sigma_{N}^{(\alpha)}=s_{1}(N)>2 H$. $N$ will be the smallest integer with this property. Then $N=N(H)$. For $N$ we have $\sigma_{N}^{(\alpha)}=s_{1}(N)>2 H$, and for any integer $p$ such that $p<N$ we have $s_{1}(p) \leqq 2 H$. If $H$ is great enough, then there exists an integer $m, m>N$, such that $\sigma_{m}^{(\alpha)}$ $\leqq(1 / 2) \cdot \sigma_{N}^{(\alpha)} . M=M(H)$ will be the smallest of these integers $m$. That is, $\sigma_{M}^{(\alpha)} \leqq(1 / 2) \cdot \sigma_{N}^{(\alpha)}$, and for $N<p<M, \sigma_{p}^{(\alpha)}>(1 / 2) \cdot \sigma_{N}^{(\alpha)}$. We shall choose $x$ by $x=x(H)=1-1 /(M \cdot N)^{1 / 2}<1 . M, N \rightarrow \infty$ with $H \rightarrow \infty$, so that $x \rightarrow 1$ with $H \rightarrow \infty$. By Lemma 4 we have $\sigma_{M}^{(\alpha)}-\sigma_{N}^{(\alpha)}$ $>-(A \cdot \ln (M / N)+1)$ or $\sigma_{N}^{(\alpha)}-\sigma_{M}^{(\alpha)}<A \cdot \ln (M / N)+1$. We also have $\sigma_{N}^{(\alpha)}-\sigma_{M}^{(\alpha)} \geqq \sigma_{N}^{(\alpha)}-(1 / 2) \cdot \sigma_{N}^{(\alpha)}=(1 / 2) \cdot \sigma_{N}^{(\alpha)}>H$, so that $M / N>e^{(H-1) / A} \rightarrow \infty$ for $H \rightarrow \infty$. Now we have

$$
\begin{array}{ll}
M \cdot(1-x)=\left(\frac{M}{N}\right)^{1 / 2} \rightarrow \infty & \text { for } H \rightarrow \infty . \\
N \cdot(1-x)=\left(\frac{N}{M}\right)^{1 / 2} \rightarrow 0 & \text { for } H \rightarrow \infty .
\end{array}
$$

By (a) there exists an infinite sequence $\left(n_{v}\right)$ such that $s_{1}\left(n_{v}\right) \geqq s_{2}\left(n_{v}\right)$. We may suppose that for $n_{\nu}, \sigma_{n_{\nu}}^{(\alpha)}=s_{1}\left(n_{\nu}\right)$ and $s_{1}\left(n_{\nu}-1\right)<\sigma_{n_{\nu}}^{(\alpha)}$. We also have $\lim _{\nu \rightarrow \infty} s_{1}\left(n_{\nu}\right)=+\infty$. Let $\epsilon_{\nu}$ be bounded and defined by $0<\epsilon_{\nu}<s_{1}\left(n_{\nu}\right)-s_{1}\left(n_{\nu}-1\right)$. We shall define $H_{\nu}$ by $H_{\nu}=(1 / 2) \cdot\left\{s_{1}\left(n_{\nu}\right)\right.$ $\left.-\epsilon_{\nu}\right\}$. Then for this sequence $\left(N_{\nu}\right)$ we have $H_{\nu} \rightarrow \infty$, because $s_{1}\left(n_{\nu}\right)$ $\rightarrow \infty$, and $\sigma_{N_{\nu}}^{(\alpha)}>2 H_{\nu}, \sigma_{r}^{(\alpha)} \leqq 2 H_{\nu}$ for $r<N_{\nu}$. By (a) and the definition of $N$ we have, for the sequence $\left(H_{\nu}\right),\left|\sigma_{r}^{(\alpha)}\right| \leqq \sigma_{N}^{(\alpha)}$ for $r<N$. Now

$$
\begin{aligned}
I & =(1-x) \cdot \sum_{n=0}^{\infty} s_{n} \cdot x^{n}=(1-x)^{\alpha+1} \cdot \sum_{n=0}^{\infty} C_{n+\alpha, n} \cdot \sigma_{n}^{(\alpha)} \cdot x^{n} \\
& =(1-x)^{\alpha+1} \cdot\left\{\sum_{0}^{N-1}+\sum_{N}^{M-1}+\sum_{M}^{\infty}\right\}=I_{1}+I_{2}+I_{3} .
\end{aligned}
$$

For the sequence $\left(H_{\nu}\right)$ we have

$$
\begin{aligned}
I_{1} & =(1-x)^{\alpha+1} \cdot \sum_{n=0}^{N-1} C_{n+\alpha, n} \cdot \sigma_{n}^{(\alpha)} \cdot x^{n}, \\
& >-s_{1}(N) \cdot(1-x)^{\alpha+1} \cdot \sum_{n=0}^{N-1} C_{n+\alpha, n} \cdot x^{n} ;
\end{aligned}
$$

by Lemma 1 and (2) it follows that $I_{1}=-\delta(H) \cdot s_{1}(n)$ where $\delta(H)$ is a function which has the limit zero for $H \rightarrow \infty$. For any integer $n$ such that $N \leqq n<M$, we have $\sigma_{n}^{(\alpha)}>(1 / 2) \cdot s_{1}(N)$, so that 


$$
\begin{aligned}
I_{2} & =(1-x)^{\alpha+1} \cdot \sum_{n=N}^{M-1} C_{n+\alpha, n} \cdot \sigma_{n}^{(\alpha)} \cdot x^{n} \\
& >\frac{1}{2} \cdot s_{1}(N) \cdot(1-x)^{\alpha+1} \cdot \sum_{n=N}^{M-1} C_{n+\alpha, n} \cdot x^{n}
\end{aligned}
$$

and by Lemma 1 it follows that $I_{2} \cong(1 / 2) \cdot s_{1}(N)$. For any integer $n$ such that $n \geqq M$, we have $\sigma_{n}^{(\alpha)}-\sigma_{M}^{(\alpha)}>-(A \cdot \ln (n / M)+1)$. We also have $\sigma_{M}^{(\alpha)}-\sigma_{M-1}^{(\alpha)}>-(A \cdot \ln (M /(M-1))+1)$. From the inequality $\sigma_{M-1}^{(\alpha)}>H$, it follows that $\sigma_{M}^{(\alpha)}>H-A \cdot \ln (M /(M-1))>1$ if $H$ is large enough. Now we have

$$
\begin{aligned}
I_{3} & =(1-x)^{\alpha+1} \cdot \sum_{n=M}^{\infty} C_{n+\alpha, n} \cdot \sigma_{n}^{(\alpha)} \cdot x^{n} \\
& =(1-x)^{\alpha+1} \cdot \sum_{n=M}^{\infty} C_{n+\alpha, n} \cdot\left\{\sigma_{M}^{(\alpha)}+\sigma_{n}^{(\alpha)}-\sigma_{M}^{(\alpha)}\right\} \cdot x^{n} \\
& >(1-x)^{\alpha+1} \cdot \sum_{n=M}^{\infty} C_{n+\alpha, n} \cdot\left(-A \cdot \ln \frac{n}{M}\right) \cdot x^{n}
\end{aligned}
$$

and by Lemma 3 and (2) we have $I_{3}>\delta(H)$, so that $I=I_{1}+I_{2}+I_{3}$ $\rightarrow \infty$ for $H_{\nu} \rightarrow \infty$, and the case (a) cannot happen.

Case (b). We have $\lim _{x+1}(1-x) \cdot \sum_{n=0}^{\infty}\left(-s_{n}\right) \cdot x^{n}=-s$. We shall define $S_{n}$ by $S_{n}=-s_{n}$, and $S_{1}(n)=\max _{r \leqq n} S_{r} ; S_{2}(n)=\max _{r \leqq n}\left(-S_{r}\right)$. It is easy to see that $S_{1}(n)=s_{2}(n)$, and $S_{2}(n)=s_{1}(n)$, so that by (b) we have for any integer $n$ greater than $n_{0}, S_{1}(n)>S_{2}(n)$. Thus the case (b) for the sequence $\left(s_{n}\right)$ is reduced, but for one reversed inequality, to the case (a) for the sequence $\left(S_{n}\right)$. As in the first part of the proof it follows, by some trivial changes, that (b) is impossible.

It follows now that $\lim \sup _{n \rightarrow \infty}\left|\sigma_{n}^{(\alpha)}\right|=L<+\infty$, so that the sequence $\left(\sigma_{n}^{(\alpha)}\right)$ is bounded. By Hardy and Littlewood's theorem it follows that $\left(s_{n}\right)$ is summable $(C, \alpha+1)$. By Theorem $2.3,\left(s_{n}\right)$ is summable $(A, \alpha)$, and the proof follows by Schmidt's theorem.

THEOREM 3.2. If the sequence $\left(s_{n}\right)$ is summable $(A, \alpha)$ for some $\alpha>-1$, and $\lim _{\lambda+1} \lim \inf _{m \rightarrow \infty} \min _{m \leqq n \leqq \lambda \cdot m}\left\{\sigma_{n}^{(\beta)}-\sigma_{m}^{(\beta)}\right\} \geqq 0$ for some $\beta \geqq \alpha$, then $\left(s_{n}\right)$ is summable $(C, \beta)$.

Proof. By Theorem 2.1 the sequence $\left(\sigma_{n_{3}}^{(\beta)}\right)$ is summable Abel, and the proof follows by Schmidt's theorem.

As a corollary of Theorems 3.1 and 3.2 we have the following corollary:

Corollary. If $\left(s_{n}\right)$ is summable Abel and for some $\alpha>-1, \lim _{\lambda+1}$ $\lim \inf _{m \rightarrow \infty} \min _{m \leqq n \leqq \lambda \cdot m}\left\{\sigma_{n}^{(\alpha)}-\sigma_{m}^{(\alpha)}\right\} \geqq 0$, then $\left(s_{n}\right)$ is summable $(C, \alpha)$. 
TheOREM 3.3. If $\left(s_{n}\right)$ is summable Abel and for some $\alpha, \alpha>-1$, the sequence $\left(\sigma_{n}^{(\alpha)}\right)$ is bounded, then $\left(s_{n}\right)$ is summable $(C, \alpha+\epsilon)$ for any $\epsilon>0$.

Andersen proved that if $\left(s_{n}\right)$ is summable $(C, \beta)$ for some $\beta>-1$, and $\sigma_{n}^{(\alpha)}=O(1)$ for some $\alpha>-1$, then $\left(s_{n}\right)$ is summable $(C, \alpha+\epsilon)$ for any $\epsilon>0$. From Theorem 2.3 it follows that Andersen's theorem is contained in Theorem 3.3.

In order to prove Theorem (3.3) we shall need the following lemma:

Lemma 5. If for some $\beta, \beta \geqq 0$, the $(C, \beta)$ means of $\left(s_{n}\right)$ are bounded, then $\lim _{\lambda \downarrow 1} \lim \sup _{m \rightarrow \infty} \max _{m \leqq n \leqq \lambda \cdot m}\left|\sigma_{n}^{(\alpha)}-\sigma_{m}^{(\alpha)}\right|=0$ for any $\alpha>\beta$.

Proof. We have

$$
\begin{aligned}
\sigma_{n}^{(\alpha)}-\sigma_{m}^{(\alpha)}= & \left(C_{n+\alpha, n}\right)^{-1} \cdot \sum_{\nu=0}^{n} C_{\alpha-\beta+n-\nu-1, n-\nu} \cdot s_{\nu}^{(\beta)}-\left(C_{m+\alpha, m}\right)^{-1} \\
& \cdot \sum_{\nu=0}^{m} C_{\alpha-\beta+m-\nu-1, m-\nu} \cdot s_{\nu}^{(\beta)} \\
= & \left(C_{n+\alpha, n}\right)^{-1} \cdot \sum_{\nu=m+1}^{n} C_{\alpha-\beta+n-\nu-1, n-\nu} \cdot C_{\beta+\nu, \nu} \cdot \sigma_{\nu}^{(\beta)} \\
& +\left(C_{n+\alpha, n}\right)^{-1} \cdot \sum_{\nu=0}^{m}\left\{C_{\alpha-\beta+n-\nu-1, n-\nu}\right. \\
& \left.-C_{\alpha-\beta+m-\nu-1, m-\nu}\right\} \cdot C_{\beta+\nu, \nu} \cdot \sigma_{\nu}^{(\beta)} \\
& -\left(C_{n+\alpha, n}\right)^{-1} \cdot\left\{C_{n+\alpha, n}-C_{m+\alpha, m}\right\} \cdot\left(C_{m+\alpha, m}\right)^{-1} \\
& \cdot \sum_{\nu=0}^{m} C_{\alpha-\beta+m-\nu-1, m-\nu} \cdot C_{\beta+\nu, \nu} \cdot \sigma_{\nu}^{(\beta)} \cdot
\end{aligned}
$$

Since $\left|\sigma_{n}^{(\beta)}\right| \leqq A$ for $n=0,1,2, \cdots$, and from the identity

$$
\begin{aligned}
& C_{\alpha+n-m-1, n-m-1} \cdot C_{\beta+m+1, m-1}+\cdots+C_{\alpha+0,0} C_{\beta+n, n} \\
& \quad=C_{\alpha+\beta+n+1, n}-C_{\alpha+\beta+m+1, m}
\end{aligned}
$$

we have

$$
\begin{aligned}
&\left|\sigma_{n}^{(\alpha)}-\sigma_{m}^{(\alpha)}\right| \leqq 2 A \cdot\left(C_{n+\alpha, n}\right)^{-1} \cdot\left\{C_{n+\alpha, n}-C_{m+\alpha, m}\right\}+A \cdot\left(C_{n+\alpha, n}\right)^{-1} \\
& \cdot\left\{ \pm\left[\sum_{\nu=0}^{m} C_{\alpha-\beta+n-\nu-1, n-\nu} \cdot C_{\beta+\nu, \nu}\right.\right. \\
&\left.\left.-\sum_{\nu=0}^{m} C_{\alpha-\beta+m-\nu-1, m-\nu} \cdot C_{\beta+\nu, \nu}\right]\right\} \\
&(+ \text { for } \alpha-\beta-1 \geqq 0,- \text { for } 0>\alpha-\beta-1>-1)
\end{aligned}
$$




$$
\begin{aligned}
\leqq & 3 A \cdot\left(C_{n+\alpha, n}\right)^{-1} \cdot\left\{C_{n+\alpha, n}-C_{m+\alpha, m}\right\} \\
& +A \cdot\left(C_{n+\alpha, n}\right)^{-1} \cdot C_{\alpha+n-m-1, n-m-1} \\
= & 3 A \cdot\left\{1-n^{\alpha} \cdot m^{-\alpha} \cdot C_{m+\alpha, m} \cdot\left(C_{n+\alpha, n}\right)^{-1} \cdot\left(\frac{m}{n}\right)^{\alpha}\right\} \\
& +\left(C_{n+\alpha, n}\right)^{-1} \cdot C_{\alpha+n-m-1, n-m-1} .
\end{aligned}
$$

For $\lambda>1$ and $m<n \leqq \lambda \cdot m$ we have now

$$
\begin{aligned}
\max _{m<n \leqq \lambda \cdot m}\left|\sigma_{n}^{(\alpha)}-\sigma_{m}^{(\alpha)}\right| & \\
\leqq & 3 A \cdot\left\{1-\min _{m<n \leqq \lambda \cdot m}\left[n^{\alpha} \cdot m^{-\alpha} \cdot C_{m+\alpha, m} \cdot\left(C_{n+\alpha, n}\right)^{-1} \cdot \lambda^{-\alpha}\right]\right\} \\
& \quad+A \cdot \max _{m<n \leqq \lambda \cdot m}\left(C_{n+\alpha, n}\right)^{-1} \cdot C_{\alpha+n-m-1, n-m-1} .
\end{aligned}
$$

Since $\lim _{n \rightarrow \infty} n^{-\alpha} \cdot C_{n+\alpha, n}=1 / \Gamma(\alpha+1)$, it follows that

$$
\begin{aligned}
\underset{m \rightarrow \infty}{\limsup } \max _{m<n \leqq \lambda \cdot m}\left|\sigma_{n}^{(\alpha)}-\sigma_{m}^{(\alpha)}\right| \\
\quad \leqq 3 A \cdot\left(1-\lambda^{-\alpha}\right)+A_{1} \cdot \lim _{m \rightarrow \infty} \operatorname{mux}_{m<n \leqq \lambda \cdot m}\left(1-\frac{m}{n}-\frac{1}{n}\right)^{\alpha} \\
\quad \leqq 3 A \cdot\left(1-\lambda^{-\alpha}\right)+A_{1} \cdot\left(1-\lambda^{-1}\right)^{\alpha},
\end{aligned}
$$

therefore

$$
\lim _{\lambda \downarrow 1} \limsup _{m \rightarrow \infty} \max _{m \leqq n \leqq \lambda \cdot m}\left|\sigma_{n}^{(\alpha)}-\sigma_{m}^{(\alpha)}\right|=0 .
$$

Q.E.D.

Proof of Theorem 3.3. By the corollary of Theorem 2.4 it follows that the sequence $\left(s_{n}\right)$ is summable $(A, \alpha+\epsilon)$ for any $\epsilon>0$. Now by Lemma 5 and Schmidt's theorem $\left(s_{n}\right)$ is summable $(C, \alpha+\epsilon)$ for any $\epsilon>0$.

4. A tauberien theorem for the Nörlund methods of summability. Let $\left(p_{n}\right), n=0,1,2, \cdots$, be a non-negative sequence such that $p_{0}>0, P_{n}=p_{0}+p_{1}+p_{2}+\cdots+p_{n} \rightarrow \infty$ and $p_{n} / P_{n} \rightarrow 0$, then we shall say that the sequence $\left(s_{n}\right)$ is summable $\left(N, p_{n}\right)$ if $\lim _{n \rightarrow \infty}\left(p_{n} \cdot s_{0}\right.$ $\left.+p_{n-1} \cdot s_{1}+\cdots+p_{0} \cdot s_{n}\right) \cdot P_{n}^{-1}$ exists. We shall define $S_{n}$ by $S_{n}=\left(p_{n} \cdot s_{0}\right.$ $\left.+\cdots+p_{0} \cdot s_{n}\right) \cdot P_{n}^{-1}$.

We shall prove the following tauberien theorem for the Nörlund methods of summability:

ThEOREM 4.1. If the sequence $\left(s_{n}\right)$ is summable $\left(N, p_{n}\right)$ to $s$ and 
$\lim _{\lambda+1} \lim \sup _{m \rightarrow \infty} \max _{m \leqq n \leqq \lambda \cdot m}\left|s_{n}-s_{m}\right|=0$, then $\lim _{n \rightarrow \infty} s_{n}=s$.

Proof. By Lemma 4 of $\$ 3$ it is easy to see that $\left|s_{n}\right| \leqq A+B$ $\cdot \ln (n+1)$, so that $\sum_{n=0}^{\infty} s_{n} \cdot x^{n}$ is convergent in the unit circle. By Cauchy's criterion for the convergence of series and the fact that $\lim _{n \rightarrow \infty} P_{n+1} / P_{n}=1$, it follows that the power series $\sum_{n=0}^{\infty} p_{n} \cdot x^{n}$ $=(1-x) \cdot \sum_{n=0}^{\infty} P_{n} \cdot x^{n}$ has the radius of convergence 1 . It is easy to see now that $\sum_{n=0}^{\infty} P_{n} \cdot S_{n} \cdot x^{n}$ is convergent in the unit circle, so that in the unit circle we have $\sum_{n=0}^{\infty} s_{n} \cdot x^{n} \cdot \sum_{n=0}^{\infty} p_{n} \cdot x^{n}=\sum_{n=0}^{\infty} P_{n} \cdot S_{n} \cdot x^{n}$. Now for $0 \leqq x<1$, we have

$$
(1-x) \cdot \sum_{n=0}^{\infty} s_{n} \cdot x^{n}=\frac{\sum_{n=0}^{\infty} P_{n} \cdot S_{n} \cdot x^{n}}{\sum_{n=0}^{\infty} P_{n} \cdot x^{n}} .
$$

It is easy to see that

$$
\lim _{x \uparrow 1} \frac{\sum_{n=0}^{\infty} P_{n} \cdot S_{n} \cdot x^{n}}{\sum_{n=0}^{\infty} P_{n} \cdot x^{n}}=s
$$

so that $\left(s_{n}\right)$ is summable Abel and the proof follows from Schmidt's theorem.

Let $k$ be some non-negative integer, and $\left(h_{n}^{(\boldsymbol{k})}\right)$ will be the Hölder means of order $k$ of the sequence $\left(s_{n}\right)$. It would be interesting to note that by using Theorem 2.1 for $\alpha=0, \beta=1 k$ times it follows that if $\left(s_{n}\right)$ is summable Abel, then $\lim _{x+1}(1-x) \cdot \sum_{n=0}^{\infty} h_{n}^{(\mathbf{k})} \cdot x^{n}=s$. From Schmidt's theorem it follows that Abel's summability of $\left(s_{n}\right)$ and $\lim _{\lambda+1} \lim \inf _{m \rightarrow \infty} \min _{m \leqq n \leqq \lambda \cdot m}\left(h_{n}^{(\boldsymbol{k})}-h_{m}^{(\mathbf{k})}\right) \geqq 0$ are sufficient for the $(H, k)$ summability of $\left(s_{n}\right)$.

The Hebrew University 